\title{
Treatment of carotid-cavernous fistula using a balloon-tipped intra-arterial catheter
}

\author{
U. M . C H O W D H A R Y \\ From the Department of Neurology, St Laurence's Hospital, Dublin, Ireland
}

SUMMARY The use of a balloon-tipped intra-arterial catheter for occlusion of the carotidcavernous fistula seems to be a simple and, by the reports published to date, very effective procedure, and its usage has been without any sequelae. Successful treatment of carotidcavernous fistulae by this method is reported in two patients.

Specific anatomical and physiological considerations along with certain pathological changes make the management of carotid-cavernous fistula a complex proposition. The fistula shunts blood from the high pressure arterial system into the low pressure venous system. Consequently, collateral vessels open, and the draining venous channels dilate.

The carotid-cavernous fistula arises from the intracavernous part of the internal carotid artery or from one of its branches, the meningohypophyseal, the capsular, and the artery to the inferior cavernous sinus, which communicate extensively with each other and provide multiple sources for the fistula. Flow from the contralateral side and from the basilar system through the circle of Willis may provide additional sources of supply.

In January 1976, when we admitted a patient with a carotid-cavernous fistula and pulsating exophthalmos, we looked for a safer and simpler surgical procedure than those used previously, and noted the technique of occlusion of the fistula by the use of a balloon-tipped intra-arterial catheter. Reports by Prolo and Hanbery (1971), Markham (1974), Picard et al. (1974), Serbinenko (1974), and Debrun et al. (1975a), showed encouraging results with this procedure.

The aim of the procedure is the occlusion of the carotid-cavernous fistula with a balloon-tipped intra-arterial catheter introduced into the lumen of the ipsilateral internal carotid artery, either through a small arteriotomy or by a percutaneous method using the Seldinger technique.

Address for reprint requests: Mr U. M. Chowdhary, Assistant in Neurosurgery, St Laurence's Hospital, Dublin 7, Ireland.

Accepted 9 June 1978

\section{Method}

The common carotid artery and its bifurcation were exposed, and an arterial clamp was placed over the external carotid artery. A small arteriotomy was made over the common carotid artery at the centre of a purse-string suture for which 5/0 Prolene was used. A size 3 or 4 Fogarty catheter (Fogarty et al., 1963) (Edward Laboratories, Santa Anna, California 92706) along with a Drum Cartridge Catheter (Abbott Laboratories, North Chicago, Illinois 60064) was introduced through this arteriotomy for a distance of $10 \mathrm{~cm}$. The tip of the drum catheter was kept $5 \mathrm{~cm}$ below the tip of the Fogarty catheter, and this was used for intraoperative carotid angiography to assess the patency of the internal carotid artery after the balloon had been inflated to close the carotidcavernous fistula.

By fluoroscopic examination the balloon of the Fogarty catheter was located. The catheter was then advanced slowly to bring the balloon to the presumed site of the fistula (Fig. 1a,b). The Fogarty balloon was then inflated slowly with radiographic contrast medium, through a threeway tap. The presence or absence of a bruit was noted. Some adjustment was needed to achieve cessation of the bruit which was regarded as evidence of occlusion of the carotid-cavernous fistula. An intraoperative angiogram was done to determine the patency of the internal carotid artery. The threeway tap was securely closed. The drum cartridge catheter was then withdrawn, the neck wound was sutured, and the proximal end of the Fogarty catheter was taped to the chest.

Periodical auscultation was carried out to detect the presence or absence of a bruit. Regular check 


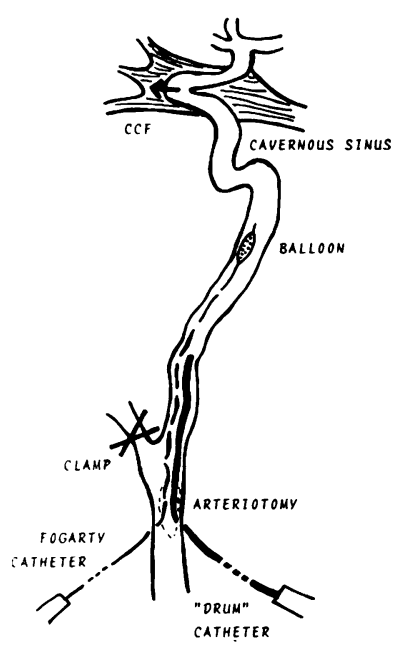

(a)

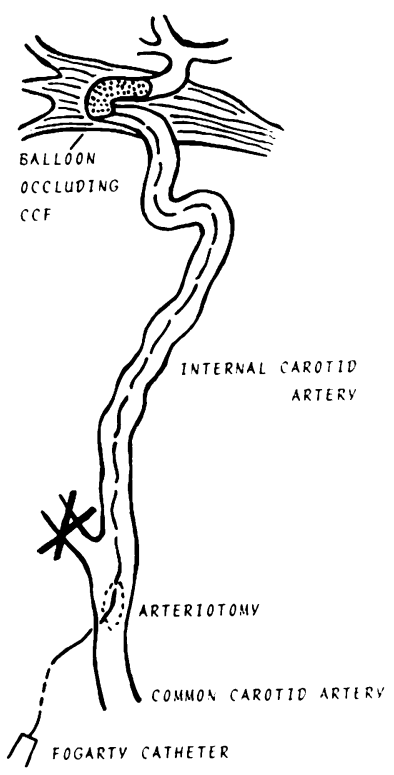

(b)
Fig. 1 Schematic drawings of the operative technique. radiographs of the skull were taken to see the position and state of inflation of the balloon. On the fifteenth day after operation the neck wound was reopened. The catheter was amputated, and its end buried.

\section{Case reports}

\section{CASE 1}

A 44 year old man (AR) was involved in an industrial accident. He regained consciousness two days later, and complained of severe constant throbbing headaches, absence of vision in the left eye, and continuous noise inside his head. A bruit could be heard over both eyes and over the left temple. External ocular movements were normal. Bilateral carotid angiography was done one week later, and showed a moderate sized left temporal intracranial extracerebral collection of blood with a traumatic dural arteriovenous fistula on the middle meningeal vessel at the level of the pterion. A carotid-cavernous fistula on the left side with retrograde filling of the superior ophthalmic vein and the pterygoid venous plexus was also shown (Fig. 2).

The left extradural haematoma was evacuated through a temporal craniectomy. The dural arteriovenous fistula was not touched. His headache and the noise inside the head persisted.

Through an arteriotomy of the left common carotid artery a number 3 Fogarty catheter was introduced and placed, under fluoroscopy, at the

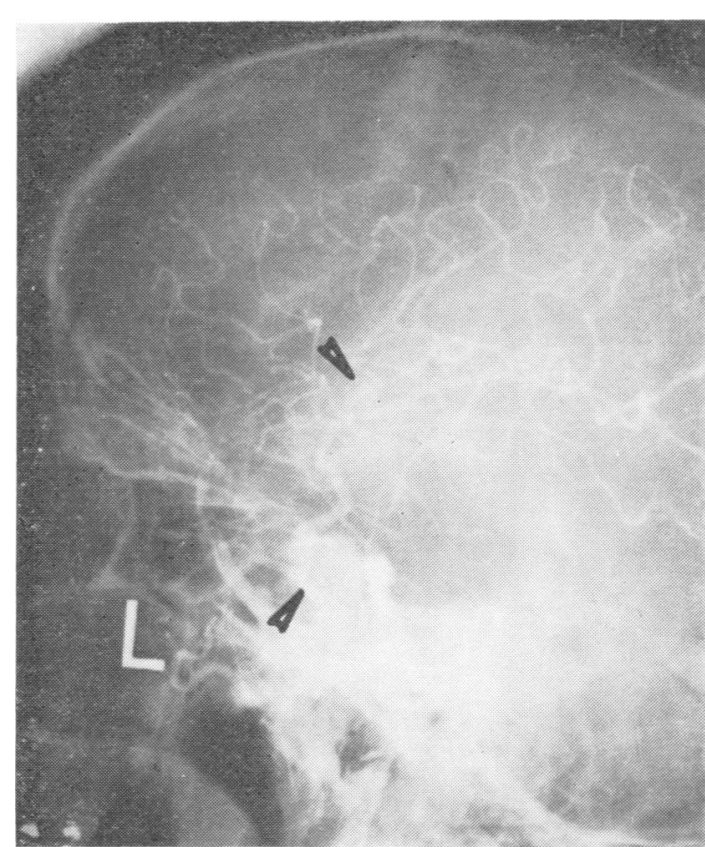

Fig. 2 Case 1. Left carotid angiograms showing the carotid-cavernous fistula and the traumatic arteriovenous fistula on the middle meningeal artery (upper arrow). 
presumed site of the carotid-cavernous fistula. This patient specifically requested general anaesthesia. With full inflation of the balloon the bruit stopped. An intraoperative angiogram showed obliteration of the lumen of the internal carotid artery and of the carotid-cavernous fistula.

For 36 hours after this procedure the patient remained well, free of headache and the noise inside the head. No bruit could be heard. The symptoms then returned, and the bruit could be heard again. Check radiography of the skull failed to show the balloon. The neck incision was reopened, and the catheter was removed. The balloon was found to have burst. The procedure was repeated with a number 4 Fogarty catheter. The carotid-cavernous fistula was obliterated successfully. The balloon also occluded the lumen of the internal carotid artery (Fig. 3). Periodical check radiographs of the skull showed continued inflation of the balloon at the original site. His headache, noise inside the head, and the bruit remained absent. On the fifteenth day after operation, the Fogarty catheter was amputated. Follow-

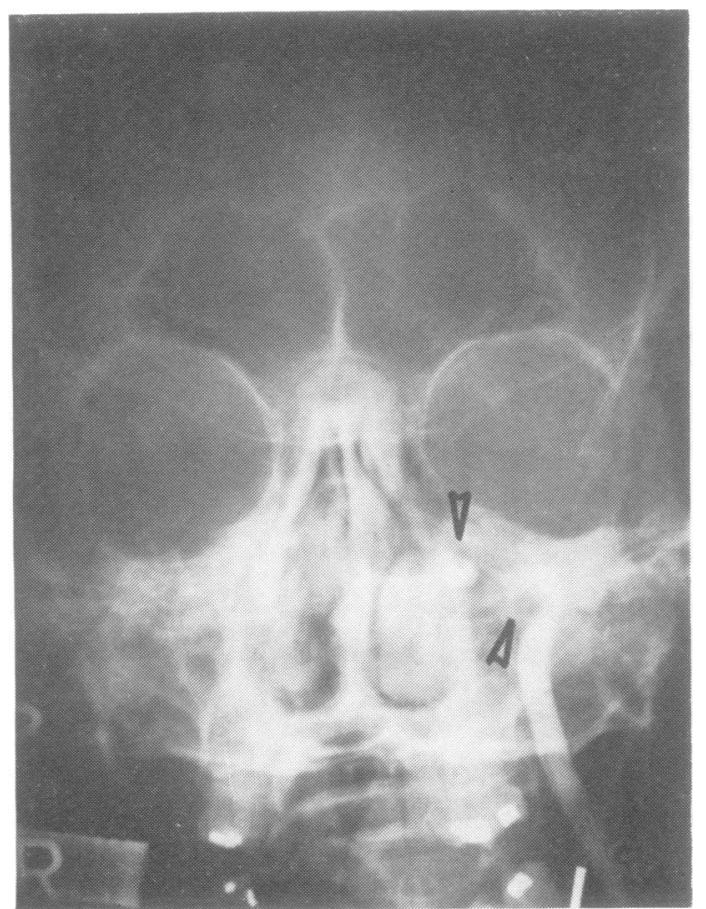

Fig. 3 Case 1. Left intraoperative carotid angiogram showing occlusion of the carotid-cavernous fistula and internal carotid artery. Upper arrow shows the contrast-filled Fogarty balloon at the site of the carotid-cavernous fistula. Lower arrow shows the occluded internal carotid artery. up studies during the last 15 months have shown him to be symptom-free and without any bruit. Radiographs of the skull taken 12 months after the procedure showed a small amount of residual dye in the balloon (Fig. 4).

\section{CASE 2}

A 39 year old man (ML) was admitted complaining of diminished vision of the right eye, noise inside the head, frequent bitemporal headaches, double vision, and loss of smell sensation for the past three years. Three years ago he had sustained injuries to the head, and was unconscious for three weeks. $\mathrm{He}$ had a past history of psychiatric illness and hospitalisation.

Neurological examination revealed anosmia, visual acuity of $6 / 9$ on the right and $6 / 6$ on the left side, proptosis of $6 \mathrm{~mm}$ on the right side with visible pulsations of the eyeball. Intraocular pressure was $26 \mathrm{mmHg}$ on the right side and $20 \mathrm{mmHg}$ on the left. The fundi showed some venous congestion on the right side. There was restriction of abduction of the right eye. A bruit was heard over both eyes, and over the right temporal region.

Bilateral carotid angiography showed a carotid 0 cavernous fistula on the right side with rapid opacification of the cavernous sinus via the proximal limb of the carotid syphon.

A size 4 Fogarty catheter was placed at the presumed site of the carotid-cavernous fistula through

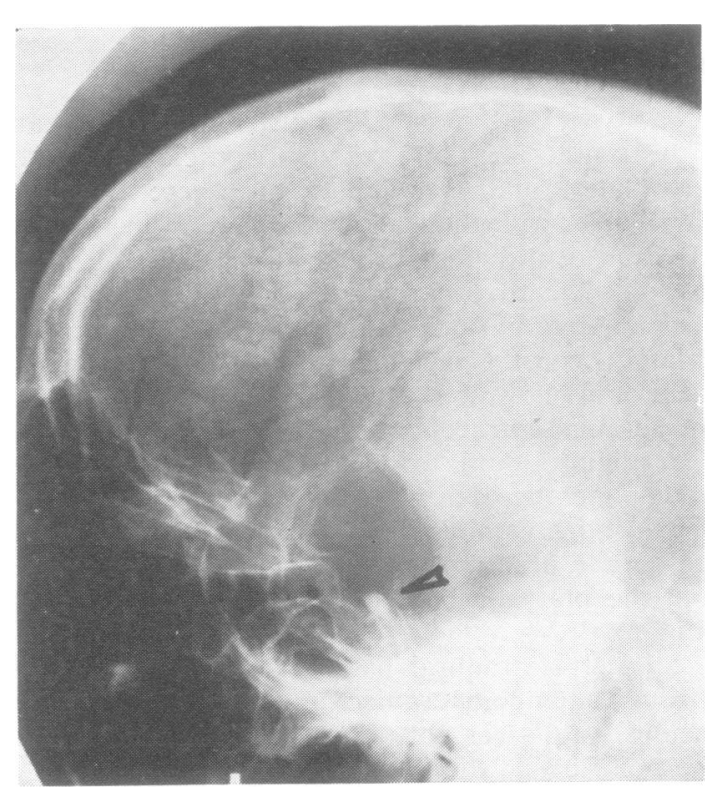

Fig. 4 Case 1. Plain lateral skull radiograph showing the residual contrast material in the Fogarty balloon 12 months after surgery. 
an arteriotomy of the right common carotid artery. The procedure was done under general anaesthesia because the patient refused to co-operate under local anaesthesia. On inflating the balloon the bruit stopped. An intraoperative right carotid angiogram showed that the internal carotid artery and the carotid-cavernous fistula were occluded. Periodical check radiographs of the skull showed continued inflation of the balloon at its original site. On the fifteenth day after operation the Fogarty catheter was amputated. The bruit, both subjectively and objectively, had remained absent all through this period. The chemosis and proptosis of the eye ceased. He acquired full ocular movement. He was discharged on the twentieth postoperative day. He failed to attend the follow-up clinic.

\section{Discussion}

Ten cases where obliteration of a carotid-cavernous fistula was done by an intra-arterial balloon catheter have been reported in the English literature. In addition, Serbinenko from Moscow, published a paper in August 1974, in which he reported treating 38 patients by this method with successful outcome in all but two patients. Details are not given in this report but the outcome is certainly impressive. In his 10 cases, and the two patients reported here, the outcome was entirely successful. The internal carotid artery was occluded in all but one. No neurological deficit was noted in any of these patients (Table).

The tolerance of an implanted Fogarty balloon has been shown in humans by Prolo and Hanbery (1971) and Debrun et al. (1975b).

Closure of a carotid-cavernous fistula with a balloon-tipped intra-arterial catheter is a simple and effective procedure. Ideally, the balloon should obliterate only the fistula and should leave the lumen of the internal carotid artery patent, but it seems that the risk of neurological deficit from obliteration of the ipsilateral carotid flow is not high. As the lumen of the ipsilateral carotid

Table Reports of treatment of carotid-cavernous fistula by balloon-tipped intra-arterial catheter

\begin{tabular}{|c|c|c|c|c|}
\hline Author & $\begin{array}{l}\text { Number } \\
\text { of cases }\end{array}$ & Result & $\begin{array}{l}\text { Carotid } \\
\text { circulation }\end{array}$ & Sequelae \\
\hline $\begin{array}{l}\text { Prolo and Hanbery (1971) } \\
\text { Markham (1974) } \\
\text { Picard et al. (1974) } \\
\text { Debrun et al. (1975a) } \\
\text { Total }\end{array}$ & $\begin{array}{r}1 \\
2 \\
5 \\
2 \\
10\end{array}$ & $\begin{array}{l}\text { Cured } \\
\text { Cured } \\
\text { Cured } \\
\text { Cured } \\
\text { Cured }\end{array}$ & $\begin{array}{l}\text { Occluded } \\
\text { Occluded } \\
\text { Occluded } \\
\text { One occluded } \\
\text { Occluded in } \\
\text { all but one }\end{array}$ & $\begin{array}{l}\text { None } \\
\text { None } \\
\text { None } \\
\text { None } \\
\text { None }\end{array}$ \\
\hline Serbinenko (1974) & 38 & $\begin{array}{l}36 \\
\text { cured }\end{array}$ & $\begin{array}{l}\text { No detail } \\
\text { available }\end{array}$ & \\
\hline
\end{tabular}

artery is occluded along with the occlusion of the fistula, "steal" of blood from the contralateral internal carotid and through the circle of Willis stops, and cerebral perfusion remains adequate. This may explain the absence of cerebral ischaemia. A further development has been reported by Debrun et al. (1975b) where they used an inflatable and released intra-arterial balloon technique to occlude the carotid-cavernous fistaula.

The procedure can be done under local anaesthesia (Picard et al., 1974; Debrun et al., 1975a) especially if the catheter is introduced percutaneously through a Seldinger needle, thus enabling monitoring of cerebral functions during the procedure. We used general anaesthesia in both patients because of special circumstances.

Introduction of the catheter through an open arteriotomy was preferred as we wanted intraoperative angiography to determine the patency of the internal carotid artery. The danger of a leaking or burst balloon is infrequent but a distinct possibility (Markham, 1974), and hence the whole of the Fogarty catheter was left in situ for 15 days, and frequent check radiographs were done to monitor the state of inflation of the balloon and its position. In our first patient the first implanted balloon burst at about 36 hours postoperatively. This was shown by the recurrence of the bruit and by the absence of the contrast medium filled balloon on the subsequent check radiograph of the skull.

I would like to thank Mr P. C. Carey and Mr J. P. Lanigan, Consultant Neurosurgeons, St Laurence's Hospital, Dublin, for allowing me to treat and report their patients.

\section{References}

Debrun, G., Lacour, P., Caron, J. P., Hurth, M., Comoy, J., Keravel, Y., and Labont, G. (1975a). Experimental approach to the treatment of carotidcavernous fistulas with an inflatable and isolated balloon. Neuroradiology, 9, 9-12.

Debrun, G., Lacour, P., Caron, J. P., Hurth, M., Comoy, J., and Keravel, Y. (1975b). Inflatable and released balloon technique: experimentation in dog -applicable in man. Neuroradiology, 9, 267-271.

Fogarty, T., Cranley, J., and Krause, R. (1963). A method for extraction of arterial emboli and thrombi. Journal of Surgery, Gynaecology, ana Obstetrics, 116, 241-244.

Luessenhop, A., and Velasquez, A. C. (1964). Observation on tolerance of intracranial arteries to catheterisation. Journal of Neurosurgery, 21, 85-91.

Markham, J. W. (1974). Carotid-cavernous fistula treated by intravascular occlusion with a balloon catheter. Journal of Neurosurgery, 40, 535-538. 
Picard, L., Lepoise, J., Montaut, J., Hepner, H., Roland, J., Guyonnaud, J. C., Jacob, F., and Andre, J. M. (1974). Endarterial occlusion of carotid-cavernous fistulas using a balloon-tipped catheter. Neuroradiology, 8, 5-10.

Prolo, D. J., and Hanbery, J. W. (1971). Intraluminal occlusion of a carotid-cavernous sinus fistula with a balloon catheter; technical note. Journal of Neurosurgery, 35, 237-242.

Serbinenko, F. A. (1974). Balloon catheterisation and occlusion of major cerebral vessels. Journal of Neurosurgery, 41, 125-145. 